\title{
Die Biedenkopf-Mitbestimmungskommission - ein Nullergebnis?
}

Michael Schumann

Reformbedarf stellt bei der deutschen Unternehmensmitbestimmung keiner in Zweifel. Arbeitgeber wie Gewerkschaften reklamieren gesetzliche Anpassungen. Das Ergebnis der Biedenkopf-Kommission legt aber überdeutlich offen: Beide Seiten verstehen darunter Nichtvereinbares. Da konnten auch die Wissenschaftler kein Unentschieden draus machen - das Spiel wurde ergebnislos abgebrochen. Alle in langen Sitzungen erarbeiteten Modernisierungsvorschläge und europatauglichen Weiterentwicklungen sind damit zunächst einmal Makulatur. Als Bedingung für einen gemeinsamen Abschluss der Kommissionsarbeit bestanden die Arbeitgeber auf der Abschaffung der Parität. Hier konnten die Gewerkschaften nicht mitmachen. Und die Wissenschaftsseite musste ihre Vermittlerrolle aufgeben.

Stellt also das Scheitern ein „Nullergebnis" (Financial Times) dar? Nicht ganz. Denn die Kommission hat in drei Punkten durchaus nützliche Klarheiten geschaffen:

1. Die akribische Auswertung der Mitbestimmungsforschung fand "keinen Nachweis negativer wirtschaftlicher Wirkungen der Unternehmensmitbestimmung“; unstrittig sei vielmehr, „dass gute Unternehmensführung die positiven Partizipationseffekte der Mitbestimmung nutzen kann". Die Wissenschaftler bestätigen: Wirtschaftlich ist Mitbestimmung eine Erfolgsgeschichte. Sie kann als komparativer Vorteil der deutschen Unternehmen gelten.

2. Für die Arbeitgeber markierte die Mitbestimmungsgesetzgebung historisch eine Niederlage. Diese Wunde ist auch über die Jahre nie geheilt. Die Position ist unverändert: Im Unternehmen wird die gesellschaftlich-wirtschaftliche Vormachtstellung der Eigentümer durch die paritätische Mitbestimmung beeinträchtigt. Alle betriebswirtschaftlichen Zugewinne im Kleinen können diesen Machtverlust im Großen nicht kompensieren. Es geht ihnen dabei um eine Klassenfrage, nicht um Jahresbilanzen.

Michael Sommer fordert die Arbeitgeber auf, „konstruktiv an der Weiterentwicklung mitzuwirken und sich nicht in die Schmollecke zurückzuziehen". Das wird ein frommer Wunsch bleiben. Denn das Scheitern einer Konsenslösung dokumentiert eine wohlüberlegte, knallharte gesellschaftspolitische Gegenposition. Weder Argumente noch Appelle versprechen da Wirkung. Die Reform der Mitbestimmung bleibt eine kontroverse Machtfrage. Deswegen konnte sie gegenwärtig nur vertagt werden. Für beide Seiten gilt: Warten auf andere Macht-Mehrheitsverhältnisse.

3. Deutlich ist auch geworden: Die Arbeitgeber bringen neue Argumente gegen die Mitbestimmung ins Spiel. Sie erschwere eine Erfolg versprechende unternehmenspolitische Antwort auf die veränderten ökonomischen Rahmenbedingungen der globalisierten Wirtschaft und der internationalisierten Kapitalmärkte. Mitbestimmung verhindere den dadurch erforderlich gewordenen Paradigmenwechsel in der deutschen Unternehmensführung. Das meint eine konsequent am Shareholder Value ausgerichtete Corporate Governance.
Und das meint eben auch: Ende der mitbestimmungsgestützten Konsenspolitik. Hohe Kapitalrendite und schnelle Gewinne erhalten entsprechend dieser unternehmenspolitischen Ausrichtung auch dann Priorität, wenn sie auf Kosten von Nachhaltigkeit und Effizienz gehen. Damit wird der Raum für tragfähige Kompromisse zwischen Kapital und Arbeit empfindlich eingeengt.

Wie sollen die Gewerkschaften mit diesen, durch die Kommissionsarbeit offengelegten „Einsichten“ umgehen?

Festzuhalten ist: Die Mitbestimmungsgesetzgebung bleibt umstrittenes Terrain. Da wird nichts geschenkt. Der Kampf um die Mitbestimmungshegemonie geht in eine neue Runde. Für die dafür notwendige gesellschaftliche Unterstützung bedeutet ein Betriebsratsskandal wie bei VW einen bitteren Rückschlag. Durch ihn konnte Mitbestimmung als „Symbol für die Verfilzung“, als „permanenter Verstoß gegen eine bestmögliche Unternehmensführung " dargestellt und in Zusammenhang mit dem Versagen der Gemeinwirtschaft gebracht werden (Frankfurter Allgemeine Zeitung, 17.01.2007). Es wäre zu einfach, die Vorfälle nur als Verfehlung von Einzelpersonen abzutun. Peter Hartz selbst sprach in seinem Prozess von fehlenden Kontrollmechanismen. Erkennbar wurden strukturelle Defizite, die es zu beheben gilt. Notwendig ist ein Mehr an Offenlegung, Begründung, Transparenz, Kontrolle. Das erfordert die Sache-aber auch der Kampf um die Köpfe. Die Gewerkschaften sollten hier durchaus selbst initiativ werden.

Gleichzeitig sollten sie aber auch inhaltlich in die Offensive gehen. Die paritätische Mitbestimmung muss in der Öffentlichkeit und für das eigene Klientel mit neuen Argumenten untermauert werden. Der von den Arbeitgebern betriebene Paradigmenwechsel in der Unternehmensführung könnte dabei ins Zentrum gerückt werden. Wenn die Arbeitgeber ihre Unternehmenspolitik einseitig am Aktionärsinteresse ausrichten wollen, dann markiert Mitbestimmung die klare Gegenposition: eine ressourcenorientierte und nachhaltige, zukunftsfähige Unternehmenspolitik, in der die Interessen der Beschäftigten gleichberechtigt mit denen des Kapitals berücksichtigt werden. Weil in Zukunft der Unternehmenserfolg mehr denn je von der bestmöglichen Nutzung und Entfaltung der Humanressourcen abhängen wird, hat die Arbeitnehmerseite mit diesem Politikansatz durchaus spielbare Karten in der Hand: durch Mitbestimmung in den Unternehmen einen neuen, zukunftsweisenden, innovativen Umgang mit der Ressource Mensch zu entwickeln. Damit könnte die paritätische Mitbestimmung ihre Legitimation nach innen und außen wirkungsvoll demonstrieren.

Michael Schumann, Prof. Dr., Präsident des Soziologischen Forschungsinstituts e. V. an der Universität Göttingen (SOFI). Arbeitsschwerpunkte: Industrie- und Arbeitssoziologie, Industrielle Beziehungen. e-mail: mschuma@gwdg.de 